\title{
Seasonal Changes of Fish Assemblages in a Subtropical Lagoon in the SE Gulf of California
}

\author{
F. Amezcua ${ }^{1}$ and F. Amezcua-Linares ${ }^{2}$ \\ ${ }^{1}$ Instituto de Ciencias del Mar y Limnología, Universidad Nacional Autónoma de México, Joel Montes Camarena S/N, \\ Mazatlán, Sin., 82040, Mexico \\ ${ }^{2}$ Instituto de Ciencias del Mar y Limnología, Universidad Nacional Autónoma de México, Circuito Exterior S/N, \\ Ciudad Universitaria, 04510, DF, Mexico
}

Correspondence should be addressed to F. Amezcua; famezcua@ola.icmyl.unam.mx

Received 28 August 2013; Accepted 30 October 2013; Published 27 January 2014

Academic Editors: P. Cardoso, J. H. Janse, P. Kaitaniemi, and M. H. Schaus

Copyright (C) 2014 F. Amezcua and F. Amezcua-Linares. This is an open access article distributed under the Creative Commons Attribution License, which permits unrestricted use, distribution, and reproduction in any medium, provided the original work is properly cited.

\begin{abstract}
The composition and seasonal changes of the fish assemblage in a coastal lagoon system in southeastern Gulf of California were assessed from December 2001 to July 2005. A total of 20,877 organisms belonging to 191 species and 47 families were analyzed. We determined that almost all the species inhabiting the system were found; however some rare species were not captured in our study. The majority of the species found were demersal but in every season at least one pelagic or benthopelagic species showed high abundances. The moonfish, Selene peruviana, was the most abundant species, whilst the puffer, Sphoeroides annulatus, was the main species in terms of biomass. The species composition changed seasonally; results from the Simpson diversity index and the cumulative species curve show that seasonally almost all the species in the system for a given season were found. These changes were also reflected in the multivariate results. The seasonal variations could be attributed to the migration of species out of the system as they grow and the arrival of new ones, which could also be related to temperature patterns since this environmental factor changes considerably through the year.
\end{abstract}

\section{Introduction}

Estuarine areas and coastal lagoons are highly productive ecosystems which are considered to be nursery areas for many invertebrate and fish species [1-4]. Coastal lagoons are also known to serve as complimentary ecosystems in the life cycle of some species because they are used as refuges for reproducing adults. In addition, the availability of food in coastal lagoons favors the development of larvae and the growth of juvenile fish $[5,6]$.

Several authors have emphasized the importance of estuaries for marine fisheries. A large part of fish landings around the world consists of species that spend at least part of their lives in estuarine waters [7-9]. Species that regularly enter the lagoon to spawn or feed and those that complete their entire life cycle in the area can be considered to be dependent on lagoon systems [10].

Lagoon systems directly support essential fisheries, with the consequence that, at present, relatively few remain unexploited [11]. Estuarine ecosystems face increased stress due to fishing activities and many estuarine habitats are being destroyed rapidly [12]. In order to understand and protect these critical habitats, it is important to document the communities they support and understand the factors that naturally influence the distribution and abundance of associated species. These environments may undergo extreme fluctuations, leading to high variability in the number and abundance of fish species [13-15].

Studies undertaken in estuaries in the temperate regions of both the northern and southern hemispheres have demonstrated cyclic, seasonal changes in fish species composition as a result of seasonal changes, as well as the migration of species which use these systems as nursery habitats, into deeper water as they increase in size $[1,16-19]$. Similar studies in tropical and subtropical estuarine habitats in south Florida, USA [20], México [21-23], Costa Rica [24], and Solomon Islands [25] have described fish assemblage distribution and structure in 
relation to seasonal variations in species number, biomass, and density and have discussed their importance as nursery areas. However, these studies fail to explain the reason of the observed changes.

Although many factors influence patterns of species composition and diversity, it has proven difficult to determine which of these covariables are most important in creating and maintaining structure within communities [26]. With the aim of addressing this question, our study describes the structure of the fish assemblage in the subtropical coastal lagoon of Santa María la Reforma (SE Gulf of California), which is one of the most important fishing grounds in the region, and its seasonal changes in relation to environmental variables. Environmental data was recorded during the surveys, which provides an opportunity to study fish assemblage patterns of diversity and abundance in relation to a suite of oceanographic variables. Specifically, water temperature, salinity, and time (months, and years) are examined in order to determine how they influence fish composition and diversity in the study system.

\section{Material and Methods}

Sampling was done at the coastal lagoon of Santa María la Reforma $\left(25^{\circ} 04^{\prime} 30^{\prime \prime} \mathrm{N}-108^{\circ} 03^{\prime} 30^{\prime \prime} \mathrm{W}\right)$ on the continental shelf of the central Mexican Pacific. The National Fisheries Institute undertook biological surveys in this lagoon at 29 stations distributed in all the different environments of the system (channels, mouth, shore, and mangroves) (Figure 1). These surveys were conducted monthly from December 2001 to May 2002, in which all stations were sampled for five consecutive days during morning hours, and seasonally from 2004 to 2005, in which all stations were sampled for one day during morning hours as well.

Because the system covers a large area, daily sampling of all stations required the use of ten $7.5 \mathrm{~m}$ boats fitted with $115 \mathrm{hp}$ outboard engines. Each boat was equipped with the three types of fishing gear commonly used in the system to catch shrimp: a shrimp trawl net with a $24 \mathrm{~m}$ footrope and a $50 \mathrm{~mm}$ liner at the codend which was used at all the stations, a $300 \mathrm{~m}$ long gill net fitted with a $75 \mathrm{~mm}$ liner which was used at all the stations, and a suripera net which is a cast net modified for trawling which was used only at the stations located in the mouth and the lagoon. A description of this fishing gear can be found in Amezcua et al. [28]. All fishing operations lasted 20 minutes and were undertaken one immediately after the other at each station before moving to the next one.

To make all tows comparable, the catch of each gear was transformed into catch-per-unit area (CPUA) estimated by dividing the total fish catch in each fishing operation by the area swept by the gear. The units were recorded as $\mathrm{kg} / \mathrm{ha}$.

To estimate the swept area, the width of each gear was recorded and then multiplied by the distance each gear was towed, estimated with the aid of a Global Positioning System (GPS). The latitude and longitude at the start and end of every fishing operation were recorded and the distance towed was

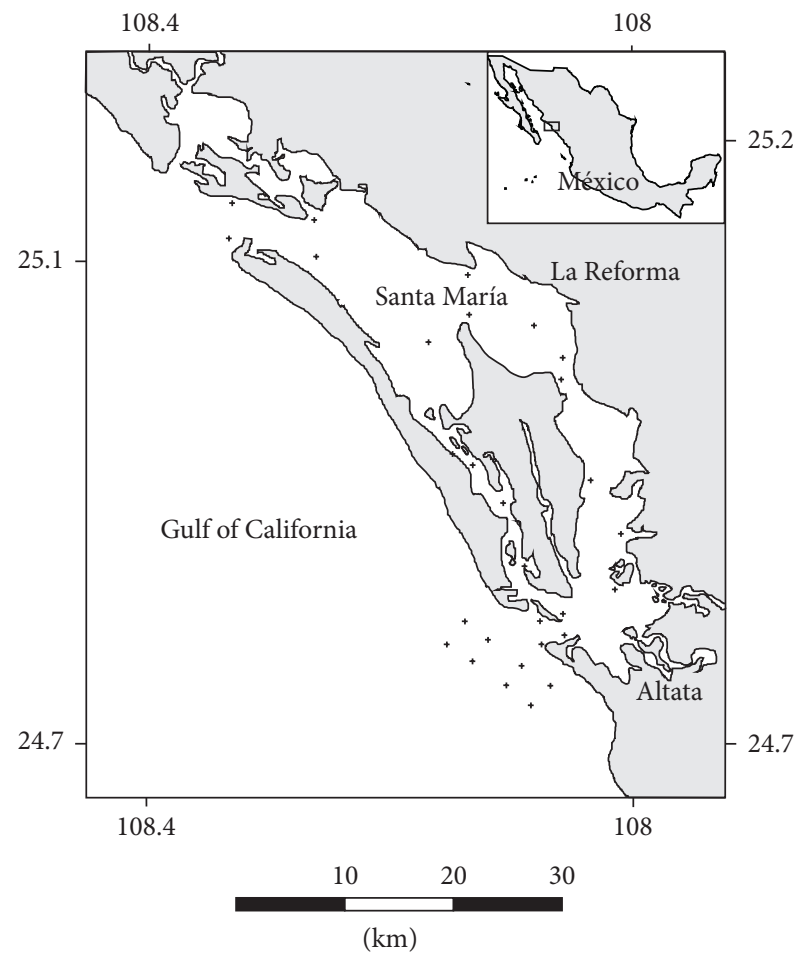

FIGURE 1: Studied area and sampling stations (dots).

estimated in nautical miles by using the equation developed by Sparre and Venema [29]:

$$
\begin{aligned}
D=60 & \left(\left(\mathrm{Lat}_{s}-\mathrm{Lat}_{e}\right)^{2}\right. \\
& \left.+\left(\operatorname{Lon}_{s}-\mathrm{Lon}_{e}\right)^{2} \cos ^{2}\left(0.5\left(\mathrm{Lat}_{s}+\mathrm{Lat}_{e}\right)\right)\right)^{1 / 2},
\end{aligned}
$$

where $D$ is the distance, $\mathrm{Lat}_{s}$ is the latitude at the start, Lat ${ }_{e}$ is the latitude at the end, Lon $_{s}$ is the longitude at the start, and Lon $_{e}$ is the longitude at the end.

This procedure was repeated for each tow with each gear, resulting in $870 \times 3$ distance records.

To standardize the different fishing gears, the area swept by every gear was derived from the cubic function of the geometry of each gear. The associated error between the sums of these areas was solved under the assumption of resolving all the possible areas of each gear to be able to integer them separately, so the area swept with each gear in each fishing operation was known. Then, the mean area swept by each gear type and its standard error were calculated using bootstrap estimates of the data and obtaining bootstrap samples which were assumed to approximate the distribution of values that would have arisen from repeatedly sampling the original sampled population. Each of these bootstrapped samples was treated as an independent random sample from the original population [30]. Two thousand independent bootstrap samples were generated. The bootstrap replicate of the parameter $\theta_{b}$ for each of the $b$ bootstrap samples 


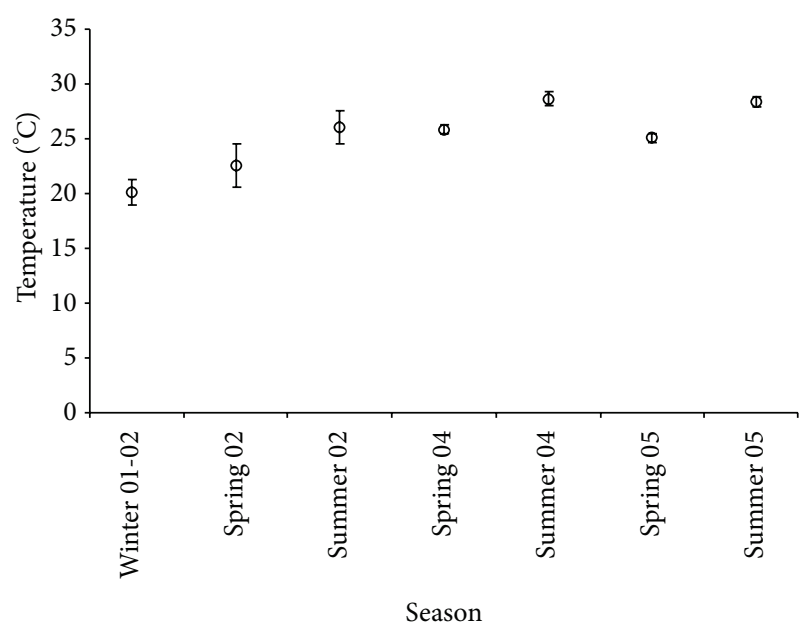

Figure 2: Mean temperature values $\left({ }^{\circ} \mathrm{C}\right)$ during the sampled seasons and years.

was calculated. The mean of the bootstrap replicates was calculated with the formula

$$
\bar{\theta}_{b}=\frac{\sum \theta_{b}}{b} .
$$

The standard error $\mathrm{se}_{\theta}$ of the parameter was estimated as

$$
\operatorname{se}_{\theta}=\sqrt{\frac{\sum\left(\theta_{b}-\bar{\theta}_{b}\right)^{2}}{b-1}}, \quad \text { [29]. }
$$

At each station, the temperature and salinity were recorded with a YSI multisensor sonde, and the fish caught by each gear were stored in plastic bags (labeled with date, station number, and the fishing gear used) and frozen. In the laboratory, fish were identified to the species level, and the total length (TL) and weight were recorded for every specimen.

Recorded temperatures were averaged seasonally and plotted into a graph to examine seasonal trends (Figure 2). The total number and biomass of fish were standardized by dividing the total fish catch in every tow by the CPUA; thus the biomass and abundance of fish per hectare were calculated. This standardized number was used for all further analyses.

The relative abundance and biomass were estimated seasonally for every species in relation to the total captured abundance and biomass, respectively [23]. Additionally, the percent occurrence of each species, defined as the proportion of months in which the species $j$ was caught, was calculated using the formula

$$
O_{i}=\frac{\text { no. of months with species } j}{\text { total no. of months }} \times 100 .
$$

A randomized cumulative species curve was constructed to determine if the number of species found in the study was close to the total number of species expected in our samples [31]. The order in which samples were analyzed was randomized 1,000 times. For each new cumulative species sample, the negative exponential model proposed for species accumulation of rare plants Magurran [32] was adjusted by minimizing the negative-logarithmic likelihood via the equation

$$
S_{t}=\beta_{0}\left(1-e^{-\beta_{1} t_{i}}\right) \text {, }
$$

where $S_{t}$ is the species richness at time $t_{i}, \beta_{0}$ is the asymptotic value of species richness $\left(S_{\max }\right)$ as $t \rightarrow+\infty$, and $\beta_{1}$ is the rate at which the maximum value is attained. For both parameters, the bias corrected percentile $95 \%$ confidence interval was calculated [30,33].

To describe the monthly species-abundance relationship, the observed data was fitted to a species-abundance model. Although species-abundance data can be described with different distributions [34], this relationship is usually examined using the following four models: (a) geometric series, (b) log series, (c) log normal, and (d) broken stick. Further details of these models can be found in Magurran [32]. To determine the goodness of fit, a Chi-Squared test of the observed and expected observations was performed. If $P<0.05$, then the distributions were significantly different at the $5 \%$ level indicating that our data did not fit that model [35]. To graphically observe the relationship, the frequency of species was plotted in relation to abundance.

Diversity was estimated using the Simpson's index $(D)$ which gives the probability that any two individuals draw at random from an infinitely large community belong to the same species. The form of the index appropriate for a finite community is

$$
D=\sum\left(\frac{n_{i}\left[n_{i}-1\right]}{N[N-1]}\right)
$$

where $n_{i}$ is the number of individuals in the $i$ th species and $N$ is the total number of individuals. As $D$ increases diversity decreases; therefore this index is usually expressed as $-\ln (D)$ following Rosenzweig [36] who explains that this transformation is easily interpretable, reflects the underlying diversity, and is independent of sample size. This index also captures the variance of the species-abundance distribution [32]. The confidence intervals for the Simpson's index were generated using a bootstrap procedure, which is a technique that allows the estimation of sample variability by resampling from the empirical probability distribution defined by a single sample. The bias corrected $95 \%$ confidence interval was obtained from 1000 bootstrap samples of species [30,33].

The fish assemblage composition was compared among the environmental factors of season (winter was defined as the period from December to February, spring from March to May, and summer from June to July; no data was available from August to November) and year using the ordination method of multidimensional scaling analysis (MDS) on Bray Curtis similarity coefficients calculated from 4th-root-transformed-abundance data. Because every season had a mean temperature, the temperature factor was included into the season factor. To test for differences in the faunal composition between the factors, an analysis of 
similarity (ANOSIM) was employed using the $R$-statistic values for pairwise comparisons to determine the degree of dissimilarity between groups [37]. Similarity of percentages (SIMPER) was used to determine which species account for most of the dissimilarities between the compositions in the different seasons and years when they were significantly different $[38,39]$. MDS was performed by Statistica 6.0 [40] from a similarity matrix obtained from PRIMER; ANOSIM and SIMPER analyses were performed by the PRIMER suite of programs [39].

\section{Results}

In total, 20,877 organisms belonging to 47 families and 191 species were analyzed (Table 1). In terms of abundance, the five most important species were Selene peruviana, which accounted for $23.08 \%$ of the total abundance, followed by Eucinostomus entomelas (7.74\%), Etropus crossotus (3.92\%), Diapterus peruvianus (3.72\%), and Eucinostomus gracilis (3.67\%). In terms of biomass, the five most important species were Sphoeroides annulatus, which accounted for $10.47 \%$ of the total biomass, followed by E. entomelas (8.92\%), Rhinobatos glaucostigma (5.11\%), Urotrygon chilensis (5.04\%), and S. peruviana (3.77\%).

The abundance and biomass of the species changed seasonally (Table 2). During winter 2001-2002 and spring 2002 the most abundant species was E. entomelas; however its abundance was much higher during winter than spring. During spring and summer 2004 the five most abundant species were the same, with similar relative abundances and a high abundance of $S$. peruviana, which accounted for approximately $50 \%$ of the total abundance in both seasons. In spring 2005, the most abundant species was Anchoa walkeri, and in summer 2005 it was Pomadasys nitidus. The most abundant species changed between 2001 and 2002 samples and 2004 and 2005 samples. While E. entomelas and D. peruvianus were characteristic of the first two seasons, the species E. gracilis, E. crossotus and P. nitidus were more characteristic of the years 2004 and 2005.

The biomass changed seasonally; during winter 2001-2002 and spring 2002 the species E. entomelas and Menticirrhus elongates were amongst the five species with the highest biomass. These results were similar to abundance results for the same time periods. S. annulatus was among the top five species in terms of biomass in all seasons of the study except for both summer seasons analyzed which were characterized by $P$. nitidus and $D$. peruvianus. S. peruviana, which was the most abundant species in both analyzed seasons of 2004, was the species with the highest biomass during spring 2004 but was not amongst the top five species with higher biomasses during the summer of the same year. Stellifer fuerthii increased its biomass from spring to summer 2004, at which point it became the species with the highest relative biomass. E. crossotus, which was highly abundant during the seasons of 2004-2005, had a high biomass only during summer 2005. In general the most abundant species also showed the highest biomasses. The species Achirus mazatlanus, D. peruvianus, E. crossotus, E. entomelas, Larimus

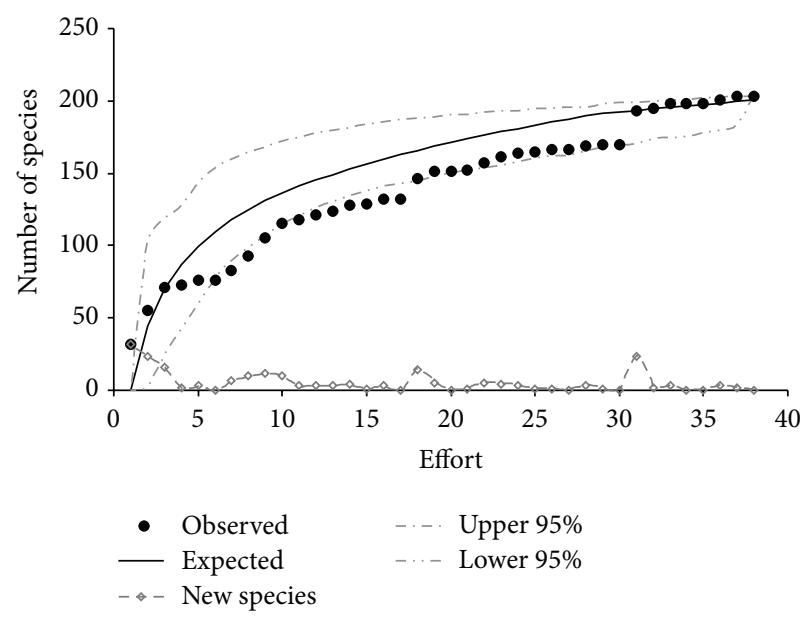

FIgURE 3: Species accumulation curve, number of new species, and expected number of species according to the von Holdridge richness model.

effulgens, Pliosteostoma lutipinnis, P. nitidus, Pseudupeneus grandisquamis, and $S$. peruviana were captured in all the sampling months; therefore their percentage of occurrence was $100 \%$.

The majority of the most abundant species, both in terms of biomass and number, were demersal organisms. A notable exception is the genus Selene spp., a benthopelagic species that showed high abundance in almost all the sampled seasons with the exception of spring 2002 and 2005, when it was not amongst the most abundant species. However, in these two seasons the pelagic species Pliosteostoma lutipinnis and Anchovia macrolepidota during spring 2002 and A. walkeri during spring 2005 showed high abundances.

Temperature and salinity data were available from all the seasons sampled as well as for summer 2002. Temperature varied seasonally, with lower temperatures occurring during winter than summer (mean values of $20^{\circ} \mathrm{C}$ and $29^{\circ} \mathrm{C}$, resp.) (Figure 2). The temperature was higher during spring and summer 2004 and 2005 than during the same seasons in 2002. Salinity was fairly uniform, ranging from 35.1 to 35.4 in all seasons and years sampled; therefore no further analyses were conducted using this factor.

The expected number of species was 200 according to the von Holdridge richness model, which is higher than the observed number of species (191). An asymptote was not reached with the species accumulation curve, indicating that some rare species were not collected (Figure 3). It is also possible to observe that throughout the study, three previous asymptotes were reached: February 2002, March 2002, and May 2004. After these months the number of new species increased.

In all months, the fish assemblage adjusted to a log normal model, indicating that there is a mode containing the midabundant species which are the majority and a small number of rare and very abundant species which are located to the left and to the right of this mode, respectively (Table 3 and Figure 4). Since very rare species are not fully represented in a finite sample, usually the left-hand tail of the distribution 
TABLE 1: Relative abundance $(A \%)$, relative biomass $(B \%)$, and frequency of occurrence $(O \%)$ of fish in Santa María la Reforma coastal lagoon, Gulf of California.

\begin{tabular}{|c|c|c|c|}
\hline Species & $A \%$ & $B \%$ & $\mathrm{O} \%$ \\
\hline \multicolumn{4}{|l|}{ Class Chondrichthyes } \\
\hline \multicolumn{4}{|l|}{ Order Torpediniformes } \\
\hline \multicolumn{4}{|l|}{ Family Narcinidae } \\
\hline Narcine entemedor Jordan and Starks, 1895 & 0.02 & 0.04 & 9.09 \\
\hline \multicolumn{4}{|l|}{ Order Rajiformes } \\
\hline \multicolumn{4}{|l|}{ Family Rhinobatidae } \\
\hline Rhinobatos glaucostigma Jordan and Gilbert, 1883 & 1.23 & 5.11 & 90.91 \\
\hline Rhinobatos productus Ayres, 1854 & 0.02 & 0.01 & 18.18 \\
\hline Zapteryx exasperata (Jordan and Gilbert, 1880) & 0.01 & 0.11 & 18.18 \\
\hline \multicolumn{4}{|l|}{ Family Dasyatidae } \\
\hline Dasyatis brevis (Garman, 1880) & 0.01 & 0.26 & 9.09 \\
\hline Dasyatis longa (Garman, 1880) & 0.08 & 0.69 & 54.55 \\
\hline \multicolumn{4}{|l|}{ Family Urolophidae } \\
\hline Urobatis halleri (Cooper, 1863) & 0.52 & 0.82 & 72.73 \\
\hline Urobatis maculatus Garman, 1913 & 0.01 & 0.02 & 18.18 \\
\hline Urotrygon aspidura (Jordan and Gilbert, 1882) & $<0.01$ & 0.01 & 9.09 \\
\hline Urotrygon chilensis (Günther, 1872) & 2.5 & 5.04 & 90.91 \\
\hline Urotrygon munda Gill, 1863 & 0.14 & 0.27 & 54.55 \\
\hline Urotrygon nana Miyake and McEachran, 1988 & 1.05 & 2.15 & 81.82 \\
\hline Urotrygon rogersi (Jordan and Starks, 1895) & 0.53 & 0.88 & 63.64 \\
\hline \multicolumn{4}{|l|}{ Family Gymnuridae } \\
\hline Gymnura marmorata (Cooper, 1864) & 0.28 & 1.47 & 63.64 \\
\hline \multicolumn{4}{|l|}{ Family Myliobatidae } \\
\hline Rhinoptera steindachneri Evermann and Jenkins, 1891 & $<0.01$ & $<0.01$ & 9.09 \\
\hline \multicolumn{4}{|l|}{ Class Actinopterygii } \\
\hline \multicolumn{4}{|l|}{ Order Albuliformes } \\
\hline \multicolumn{4}{|l|}{ Family Albulidae } \\
\hline Albula nemoptera (Fowler, 1911) & 1 & 2.78 & 54.55 \\
\hline Albula vulpes (Linnaeus, 1758) & 0.85 & 2.46 & 54.55 \\
\hline \multicolumn{4}{|l|}{ Order Anguilliformes } \\
\hline \multicolumn{4}{|l|}{ Family Muraenidae } \\
\hline Gymnothorax panamensis (Steindachner, 1876) & 0.11 & 0.51 & 54.55 \\
\hline \multicolumn{4}{|l|}{ Family Congridae } \\
\hline Rhynchoconger nitens (Jordan and Bollman, 1890) & 0.05 & 0.09 & 27.27 \\
\hline \multicolumn{4}{|l|}{ Family Ophichthidae } \\
\hline Bascanichthys panamensis Meek and Hildebrand, 1923 & 0.01 & 0.1 & 9.09 \\
\hline Echiophis brunneus (Castro-Aguirre and Suárez de los Cobos, 1983) & $<0.01$ & $<0.01$ & 9.09 \\
\hline Ophichthus zophochir Jordan and Gilbert, 1882 & 0.02 & 0.04 & 27.27 \\
\hline Pseudomyrophis micropinna Wade, 1946 & $<0.01$ & $<0.01$ & 9.09 \\
\hline \multicolumn{4}{|l|}{ Order Clupeiformes } \\
\hline \multicolumn{4}{|l|}{ Family Pristigasteridae } \\
\hline Pliosteostoma lutipinnis (Jordan and Gilbert, 1882) & 1.82 & 1.29 & 100.00 \\
\hline Opisthopterus dovii (Günther, 1868) & 0.44 & 0.23 & 54.55 \\
\hline \multicolumn{4}{|l|}{ Family Engraulidae } \\
\hline Anchoa argentivittata (Regan, 1904) & 0.01 & $<0.01$ & 18.18 \\
\hline Anchoa helleri (Hubbs, 1921) & 0.11 & 0.03 & 36.36 \\
\hline Anchoa mundeola (Gilbert and Pierson, 1898) & 0.28 & 0.08 & 36.36 \\
\hline
\end{tabular}


TABle 1: Continued.

\begin{tabular}{|c|c|c|c|}
\hline Species & $A \%$ & $B \%$ & $\mathrm{O} \%$ \\
\hline Anchoa nasus (Kner and Steindachner, 1867) & 0.37 & 0.11 & 54.55 \\
\hline Anchoa walkeri Baldwin and Chang, 1970 & 1.45 & 0.45 & 63.64 \\
\hline Anchovia macrolepidota (Kner, 1863) & 1.17 & 0.57 & 72.73 \\
\hline Cetengraulis mysticetus (Günther, 1867) & 0.08 & 0.03 & 18.18 \\
\hline Engraulis mordax Girard, 1854 & 0.02 & $<0.01$ & 9.09 \\
\hline \multicolumn{4}{|l|}{ Family Clupeidae } \\
\hline Lile stolifera (Jordan and Gilbert, 1882) & 0.01 & 0.39 & 27.27 \\
\hline Opisthonema libertate (Günther, 1867) & 0.23 & 0.31 & 54.55 \\
\hline Opisthonema medirastre Berry and Barrett, 1963 & 0.14 & 0.15 & 36.36 \\
\hline \multicolumn{4}{|l|}{ Order Siluriformes } \\
\hline \multicolumn{4}{|l|}{ Family Ariidae } \\
\hline Ariopsis guatemalensis (Günther, 1864) & 0.01 & 0.03 & 18.18 \\
\hline Ariopsis seemanni (Günther, 1864) & 0.16 & 0.36 & 18.18 \\
\hline Cathorops dasycephalus Günther, 1864 & $<0.01$ & 0.01 & 9.09 \\
\hline Occidentarius platypogon (Günther, 1864) & 0.26 & 0.58 & 54.55 \\
\hline Bagre panamensis Gill, 1863 & 0.28 & 0.43 & 63.64 \\
\hline Cathorops liropus (Bristol, 1896) & 0.07 & 0.07 & 36.36 \\
\hline Notarius troschelii (Gill, 1863) & 0.03 & 0.01 & 9.09 \\
\hline \multicolumn{4}{|l|}{ Order Aulopiformes } \\
\hline \multicolumn{4}{|l|}{ Family Synodontidae } \\
\hline Synodus evermanni Jordan and Bollman, 1890 & $<0.01$ & $<0.01$ & 9.09 \\
\hline Synodus scituliceps Jordan and Gilbert, 1882 & 0.88 & 1.85 & 90.91 \\
\hline \multicolumn{4}{|l|}{ Order Ophidiiformes } \\
\hline \multicolumn{4}{|l|}{ Family Ophidiidae } \\
\hline Lepophidium pardale (Gilbert, 1890) & 0.02 & 0.03 & 9.09 \\
\hline Lepophidium prorates (Jordan and Bollman, 1890) & 0.02 & 0.02 & 18.18 \\
\hline Otophidium indefatigabile Jordan and Bollman, 1890 & $<0.01$ & $<0.01$ & 18.18 \\
\hline \multicolumn{4}{|l|}{ Order Batrachoidiformes } \\
\hline \multicolumn{4}{|l|}{ Family Batrachoididae } \\
\hline Porichthys analis Hubbs and Schultz, 1939 & 0.55 & 0.65 & 54.55 \\
\hline \multicolumn{4}{|l|}{ Order Lophiiformes } \\
\hline \multicolumn{4}{|l|}{ Family Lophiidae } \\
\hline Lophiodes caulinaris (Garman, 1899) & 0.1 & 0.04 & 27.27 \\
\hline \multicolumn{4}{|l|}{ Family Antennariidae } \\
\hline Antennarius avalonis Jordan and Starks, 1907 & 0.07 & 0.03 & 18.18 \\
\hline \multicolumn{4}{|l|}{ Order Beloniformes } \\
\hline \multicolumn{4}{|l|}{ Family Hemiramphidae } \\
\hline Hemiramphus saltator Gilbert and Starks, 1904 & $<0.01$ & $<0.01$ & 9.09 \\
\hline \multicolumn{4}{|l|}{ Order Syngnathiformes } \\
\hline \multicolumn{4}{|l|}{ Family Fistulariidae } \\
\hline Fistularia corneta Gilbert and Starks, 1904 & 0.02 & 0.01 & 27.27 \\
\hline \multicolumn{4}{|l|}{ Family Syngnathidae } \\
\hline Hippocampus ingens Girard, 1858 & 0.04 & 0.01 & 45.45 \\
\hline \multicolumn{4}{|l|}{ Order Scorpaeniformes } \\
\hline \multicolumn{4}{|l|}{ Family Scorpaenidae } \\
\hline Pontinus sierra (Gilbert, 1890) & 0.1 & 0.03 & 54.55 \\
\hline Scorpaena mystes Jordan and Starks, 1895 & 0.1 & 0.05 & 18.18 \\
\hline Scorpaena sonorae Jenkins and Evermann, 1889 & 0.1 & $<0.01$ & 9.09 \\
\hline
\end{tabular}


TABle 1: Continued.

\begin{tabular}{|c|c|c|c|}
\hline Species & $A \%$ & $B \%$ & $\mathrm{O} \%$ \\
\hline \multicolumn{4}{|l|}{ Family Triglidae } \\
\hline Bellator loxias (Jordan, 1897) & 0.03 & 0.01 & 9.09 \\
\hline Bellator xenisma (Jordan and Bollman, 1890) & 0.2 & 0.16 & 63.64 \\
\hline Prionotus albirostris Jordan and Bollman, 1890 & 0.01 & $<0.01$ & 18.18 \\
\hline Prionotus birostratus Richardson, 1844 & $<0.01$ & 0.01 & 9.09 \\
\hline Prionotus horrens Richardson, 1844 & 0.13 & 0.04 & 27.27 \\
\hline Prionotus ruscarius Gilbert and Starks, 1904 & 0.28 & 0.44 & 63.64 \\
\hline Prionotus stephanophrys Lockington, 1881 & 0.49 & 0.32 & 63.64 \\
\hline \multicolumn{4}{|l|}{ Order Perciformes } \\
\hline \multicolumn{4}{|l|}{ Family Centropomidae } \\
\hline Centropomus nigrescens Günther, 1864 & $<0.01$ & 0.01 & 9.09 \\
\hline Centropomus robalito Jordan and Gilbert, 1882 & 0.06 & 0.07 & 45.45 \\
\hline \multicolumn{4}{|l|}{ Family Serranidae } \\
\hline Diplectrum eumelum Rosenblatt and Johnson, 1974 & 0.22 & 0.37 & 36.36 \\
\hline Diplectrum euryplectrum Jordan and Bollman, 1890 & 0.08 & 0.12 & 27.27 \\
\hline Diplectrum labarum Rosenblatt and Johnson, 1974 & 0.01 & 0.01 & 18.18 \\
\hline Diplectrum macropoma (Günther, 1864) & 0.09 & 0.07 & 18.18 \\
\hline Diplectrum pacificum Meek and Hildebrand, 1925 & 0.2 & 0.29 & 45.45 \\
\hline Diplectrum rostrum Bortone, 1974 & 0.02 & 0.03 & 9.09 \\
\hline Diplectrum sciurus Gilbert, 1892 & 0.01 & 0.01 & 9.09 \\
\hline Epinephelus analogus Gill, 1863 & 0.21 & 0.41 & 54.55 \\
\hline Epinephelus exsul (Fowler, 1944) & 0.01 & 0.01 & 9.09 \\
\hline Mycteroperca rosacea (Streets, 1877 ) & $<0.01$ & 0.01 & 9.09 \\
\hline Paralabrax maculatofasciatus (Steindachner, 1868) & 0.11 & 0.21 & 54.55 \\
\hline \multicolumn{4}{|l|}{ Family Nematistiidae } \\
\hline Nematistius pectoralis Gill, 1862 & 0.19 & 0.59 & 18.18 \\
\hline \multicolumn{4}{|l|}{ Family Carangidae } \\
\hline Carangoides otrynter (Jordan and Gilbert, 1883) & 0.1 & 0.13 & 54.55 \\
\hline Caranx caballus Günther, 1868 & 0.23 & 0.37 & 45.45 \\
\hline Caranx caninus (Günther, 1867) & 0.49 & 0.79 & 54.55 \\
\hline Caranx vinctus Jordan and Gilbert, 1882 & 0.29 & 0.41 & 63.64 \\
\hline Chloroscombrus orqueta Jordan and Gilbert, 1883 & 0.2 & 0.12 & 45.45 \\
\hline Decapterus muroadsi (Temminck and Schlegel, 1844) & $<0.01$ & $<0.01$ & 9.09 \\
\hline Hemicaranx leucurus (Günther, 1864) & 0.05 & 0.06 & 27.27 \\
\hline Hemicaranx zelotes Gilbert, 1898 & 0.03 & 0.05 & 27.27 \\
\hline Oligoplites altus (Günther, 1868) & 0.46 & 0.91 & 36.36 \\
\hline Oligoplites refulgens Gilbert and Starks, 1904 & 0.11 & 0.10 & 63.64 \\
\hline Oligoplites saurus (Bloch and Schneider, 1801) & 0.11 & 0.22 & 27.27 \\
\hline Selar crumenophthalmus (Bloch, 1793) & 0.13 & 0.33 & 27.27 \\
\hline Selene brevoortii (Gill, 1863) & 2.29 & 1.05 & 81.82 \\
\hline Selene oerstedii Lütken, 1880 & 0.02 & 0.01 & 18.18 \\
\hline Selene peruviana (Guichenot, 1866) & 23.08 & 3.77 & 100.00 \\
\hline Trachinotus kennedyi Steindachner, 1876 & 0.07 & 0.16 & 45.45 \\
\hline Trachinotus paitensis Cuvier, 1832 & 0.04 & 0.09 & 27.27 \\
\hline \multicolumn{4}{|l|}{ Family Lutjanidae } \\
\hline Hoplopagrus guentherii Gill, 1862 & 0.02 & 0.08 & 9.09 \\
\hline Lutjanus argentiventris (Peters, 1869) & 0.01 & 0.02 & 18.18 \\
\hline Lutjanus guttatus (Steindachner, 1869) & 0.12 & 0.18 & 45.45 \\
\hline
\end{tabular}


TABle 1: Continued.

\begin{tabular}{|c|c|c|c|}
\hline Species & $A \%$ & $B \%$ & $\mathrm{O} \%$ \\
\hline Lutjanus novemfasciatus Gill, 1862 & $<0.01$ & $<0.01$ & 9.09 \\
\hline \multicolumn{4}{|l|}{ Family Gerreidae } \\
\hline Diapterus aureolus (Jordan and Gilbert, 1882) & 0.13 & 0.04 & 18.18 \\
\hline Diapterus peruvianus (Cuvier, 1830) & 3.72 & 3.62 & 100.00 \\
\hline Eucinostomus argenteus Baird and Girard, 1855 & 1.16 & 1.36 & 63.64 \\
\hline Eucinostomus currani Zauranec, 1980 & 0.97 & 0.62 & 72.73 \\
\hline Eucinostomus entomelas Zauranec, 1980 & 7.76 & 8.99 & 100.00 \\
\hline Eucinostomus gracilis (Gill, 1862) & 3.67 & 1.57 & 72.73 \\
\hline Eugerres axillaris (Günther, 1864) & 0.1 & 0.05 & 9.09 \\
\hline Eugerres lineatus (Humboldt, 1821) & $<0.01$ & $<0.01$ & 9.09 \\
\hline Gerres cinereus (Walbaum, 1792) & 0.09 & 0.06 & 36.36 \\
\hline \multicolumn{4}{|l|}{ Family Haemulidae } \\
\hline Conodon serrifer Jordan and Gilbert, 1882 & 0.04 & 0.06 & 45.45 \\
\hline Haemulon scudderii Gill, 1862 & 0.09 & 0.15 & 27.27 \\
\hline Haemulon sexfasciatum Gill, 1862 & 0.06 & 0.09 & 18.18 \\
\hline Haemulopsis elongatus (Steindachner, 1879) & 0.01 & 0.01 & 9.09 \\
\hline Haemulopsis leuciscus (Günther, 1864) & 0.15 & 0.27 & 45.45 \\
\hline Haemulopsis nitidus (Steindachner, 1869) & 0.46 & 0.19 & 18.18 \\
\hline Microlepidotus brevipinnis (Steindachner, 1869) & 0.39 & 0.23 & 27.27 \\
\hline Orthopristis cantharinus (Jenyns, 1840) & 0.07 & 0.11 & 36.36 \\
\hline Orthopristis chalceus (Günther, 1864) & 0.03 & 0.05 & 36.36 \\
\hline Orthopristis reddingi Jordan and Richardson, 1895 & 0.22 & 0.39 & 36.36 \\
\hline Haemulopsis axillaris (Steindachner, 1869) & 0.07 & 0.04 & 36.36 \\
\hline Pomadasys branickii (Steindachner, 1879) & 0.51 & 0.73 & 81.82 \\
\hline Haemulopsis elongatus (Steindachner, 1879) & 0.27 & 0.35 & 54.55 \\
\hline Haemulopsis leuciscus (Günther, 1864) & 0.36 & 0.81 & 63.64 \\
\hline Pomadasys macracanthus (Günther, 1864) & 0.02 & 0.05 & 18.18 \\
\hline Haemulopsis nitidus (Steindachner, 1869) & 2.86 & 2.51 & 100.00 \\
\hline Pomadasys panamensis (Steindachner, 1876) & 2.77 & 2.80 & 90.91 \\
\hline \multicolumn{4}{|l|}{ Family Polynemidae } \\
\hline Polydactylus approximans (Lay and Bennett, 1839) & 0.51 & 0.39 & 81.82 \\
\hline \multicolumn{4}{|l|}{ Family Sciaenidae } \\
\hline Bairdiella icistia (Jordan and Gilbert, 1882) & 0.01 & $<0.01$ & 18.18 \\
\hline Corvula macrops (Steindachner, 1876) & 0.03 & 0.01 & 9.09 \\
\hline Cynoscion reticulatus (Günther, 1864) & 0.27 & 0.62 & 81.82 \\
\hline Cynoscion parvipinnis Ayres, 1861 & 0.03 & 0.13 & 18.18 \\
\hline Cynoscion stolzmanni (Steindachner, 1879) & 0.1 & 0.20 & 27.27 \\
\hline Elattarchus archidium (Jordan and Gilbert, 1882) & 0.09 & 0.08 & 45.45 \\
\hline Isopisthus remifer Jordan and Gilbert, 1882 & 0.25 & 0.45 & 63.64 \\
\hline Larimus acclivis Jordan and Bristol, 1898 & 0.44 & 0.28 & 72.73 \\
\hline Larimus argenteus (Gill, 1863) & 0.06 & 0.04 & 18.18 \\
\hline Larimus effulgens Gilbert, 1898 & 0.68 & 0.68 & 100.00 \\
\hline Larimus pacificus Jordan and Bollman, 1890 & 0.24 & 0.12 & 54.55 \\
\hline Menticirrhus elongatus (Günther, 1864) & 0.9 & 3.31 & 54.55 \\
\hline Menticirrhus nasus (Günther, 1868) & 0.2 & 0.55 & 72.73 \\
\hline Menticirrhus panamensis (Steindachner, 1876) & 0.04 & 0.10 & 27.27 \\
\hline Micropogonias altipinnis (Günther, 1864) & 0.13 & 0.09 & 27.27 \\
\hline Ophioscion imiceps (Jordan and Gilbert, 1882) & 0.09 & 0.10 & 18.18 \\
\hline
\end{tabular}


TABle 1: Continued.

\begin{tabular}{|c|c|c|c|}
\hline Species & $A \%$ & $B \%$ & $\mathrm{O} \%$ \\
\hline Ophioscion strabo Gilbert, 1897 & 0.08 & 0.11 & 45.45 \\
\hline Stellifer ericymba (Jordan and Gilbert, 1882) & 0.02 & 0.04 & 27.27 \\
\hline Stellifer fuerthii (Steindachner, 1876) & 2.21 & 1.17 & 63.64 \\
\hline Stellifer illecebrosus Gilbert, 1898 & 0.29 & 0.22 & 54.55 \\
\hline Umbrina xanti Gill, 1862 & 0.01 & 0.04 & 18.18 \\
\hline \multicolumn{4}{|l|}{ Family Mullidae } \\
\hline Mulloidichthys dentatus (Gill, 1862) & 0.06 & 0.05 & 18.18 \\
\hline Pseudupeneus grandisquamis (Gill, 1863) & 1.14 & 0.64 & 100.00 \\
\hline \multicolumn{4}{|l|}{ Family Mugilidae } \\
\hline Mugil cephalus Linnaeus, 1758 & 0.11 & 0.2 & 36.36 \\
\hline Mugil curema Valenciennes, 1836 & 0.11 & 0.28 & 54.55 \\
\hline \multicolumn{4}{|l|}{ Family Ephippidae } \\
\hline Chaetodipterus zonatus (Girard, 1858) & 1.36 & 1.2 & 90.91 \\
\hline Parapsettus panamensis (Steindachner, 1876) & $<0.01$ & $<0.01$ & 9.09 \\
\hline \multicolumn{4}{|l|}{ Family Chaetodontidae } \\
\hline Chaetodon humeralis Günther, 1860 & 0.11 & 0.02 & 36.36 \\
\hline \multicolumn{4}{|l|}{ Family Pomacanthidae } \\
\hline Pomacanthus zonipectus (Gill, 1862) & $<0.01$ & 0.01 & 9.09 \\
\hline \multicolumn{4}{|l|}{ Family Sphyraenidae } \\
\hline Sphyraena ensis Jordan and Gilbert, 1882 & 0.06 & 0.09 & 45.45 \\
\hline \multicolumn{4}{|l|}{ Family Uranoscopidae } \\
\hline Kathetostoma averruncus Jordan and Bollman, 1890 & 0.04 & 0.03 & 18.18 \\
\hline \multicolumn{4}{|l|}{ Family Gobiidae } \\
\hline Bollmannia chlamydes Jordan, 1890 & $<0.01$ & $<0.01$ & 9.09 \\
\hline \multicolumn{4}{|l|}{ Family Trichiuridae } \\
\hline Trichiurus nitens Garman, 1899 & 0.09 & 0.08 & 9.09 \\
\hline \multicolumn{4}{|l|}{ Family Scombridae } \\
\hline Scomber japonicus Houttuyn, 1782 & 0.05 & 0.28 & 27.27 \\
\hline Scomberomorus sierra Jordan and Starks, 1895 & 0.63 & 2.03 & 54.55 \\
\hline \multicolumn{4}{|l|}{ Family Stromateidae } \\
\hline Peprilus medius (Peters, 1869) & 0.25 & 0.45 & 63.64 \\
\hline Peprilus simillimus (Ayres, 1860) & 0.01 & 0.01 & 9.09 \\
\hline Peprilus snyderi Gilbert and Starks, 1904 & 0.09 & 0.14 & 63.64 \\
\hline \multicolumn{4}{|l|}{ Order Pleuronectiformes } \\
\hline \multicolumn{4}{|l|}{ Family Bothidae } \\
\hline Bothus constellatus (Jordan, 1889) & 0.09 & 0.08 & 36.36 \\
\hline \multicolumn{4}{|l|}{ Family Paralichthyidae } \\
\hline Ancylopsetta dendritica Gilbert, 1890 & 0.01 & 0.01 & 18.18 \\
\hline Citharichthys fragilis Gilbert, 1890 & $<0.01$ & $<0.01$ & 9.09 \\
\hline Citharichthys gilberti Jenkins and Evermann, 1889 & 0.68 & 0.34 & 81.82 \\
\hline Citharichthys platophrys Gilbert, 1891 & 0.01 & 0.01 & 9.09 \\
\hline Citharichthys xanthostigma Gilbert, 1890 & 0.03 & 0.01 & 9.09 \\
\hline Cyclopsetta panamensis (Steindachner, 1876) & 0.55 & 0.65 & 72.73 \\
\hline Cyclopsetta querna (Jordan and Bollman, 1890) & 0.25 & 0.47 & 81.82 \\
\hline Etropus crossotus Jordan and Gilbert, 1882 & 4.30 & 1.80 & 100.00 \\
\hline Hippoglossina bollmani Gilbert, 1890 & 0.02 & 0.04 & 9.09 \\
\hline Paralichthys woolmani Jordan and Williams, 1897 & 0.02 & 0.06 & 36.36 \\
\hline Syacium latifrons (Jordan and Gilbert, 1882) & 0.01 & 0.01 & 9.09 \\
\hline
\end{tabular}


TABle 1: Continued.

\begin{tabular}{|c|c|c|c|}
\hline Species & $A \%$ & $B \%$ & $\mathrm{O} \%$ \\
\hline Syacium ovale (Günther, 1864) & 2.16 & 1.20 & 81.82 \\
\hline \multicolumn{4}{|l|}{ Family Achiridae } \\
\hline Achirus mazatlanus (Steindachner, 1869) & 1.3 & 0.65 & 100.00 \\
\hline Trinectes fonsecensis (Günther, 1862) & 0.01 & $<0.01$ & 9.09 \\
\hline \multicolumn{4}{|l|}{ Family Cynoglossidae } \\
\hline Symphurus atramentatus Jordan and Bollman, 1890 & 0.01 & 0.01 & 18.18 \\
\hline Symphurus atricaudus (Jordan and Gilbert, 1880) & 0.03 & 0.01 & 9.09 \\
\hline Symphurus elongatus (Günther, 1868) & 0.33 & 0.13 & 27.27 \\
\hline Symphurus leei Jordan and Bollman, 1890 & 0.01 & 0.01 & 9.09 \\
\hline Symphurus melanurus Clark, 1936 & 0.17 & 0.07 & 9.09 \\
\hline Symphurus prolatinaris Munroe, Nizinski, and Mahadeva, 1991 & 0.01 & 0.01 & 9.09 \\
\hline \multicolumn{4}{|l|}{ Order Tetraodontiformes } \\
\hline \multicolumn{4}{|l|}{ Family Balistidae } \\
\hline Balistes polylepis Steindachner, 1876 & 0.37 & 0.5 & 72.73 \\
\hline Pseudobalistes naufragium (Jordan and Starks, 1895) & 0.02 & $<0.01$ & 18.18 \\
\hline \multicolumn{4}{|l|}{ Family Tetraodontidae } \\
\hline Canthigaster punctatissima (Günther, 1870) & 0.01 & $<0.01$ & 9.09 \\
\hline Sphoeroides annulatus (Jenyns, 1842) & 3.33 & 10.54 & 81.82 \\
\hline Sphoeroides lobatus (Steindachner, 1870) & 1.28 & 0.83 & 81.82 \\
\hline
\end{tabular}

TABle 2: Percentage of the top five species in terms of abundance and biomass in each sampled season and its known habitat (H) [27]

\begin{tabular}{|c|c|c|c|c|c|c|c|c|}
\hline \multicolumn{9}{|c|}{ Abundance } \\
\hline Winter 2001-2002 & $\%$ & $\mathrm{H}$ & Spring 2002 & $\%$ & $\mathrm{H}$ & Spring 2004 & $\%$ & $\mathrm{H}$ \\
\hline Eucinostomus entomelas & 22 & $\mathrm{D}$ & Eucinostomus entomelas & 6 & $\mathrm{D}$ & Selene peruviana & 53 & B \\
\hline Sphoeroides annulatus & 8 & $\mathrm{D}$ & Pliosteostoma lutipinnis & 5 & $\mathrm{P}$ & Eucinostomus gracilis & 7 & $\mathrm{D}$ \\
\hline Selene brevoortii & 8 & $\mathrm{~B}$ & Diapterus peruvianus & 5 & $\mathrm{D}$ & Etropus crossotus & 6 & $\mathrm{D}$ \\
\hline Diapterus peruvianus & 6 & $\mathrm{D}$ & Anchovia macrolepidota & 4 & $\mathrm{P}$ & Stellifer fuerthii & 4 & $\mathrm{D}$ \\
\hline Urotrygon chilensis & 4 & $\mathrm{D}$ & Pomadasys panamensis & 4 & $\mathrm{D}$ & Pomadasys nitidus & 3 & $\mathrm{D}$ \\
\hline Summer 2004 & $\%$ & $\mathrm{H}$ & Spring 2005 & $\%$ & $\mathrm{H}$ & Summer 2005 & $\%$ & $\mathrm{H}$ \\
\hline Selene peruviana & 50 & $\mathrm{~B}$ & Anchoa walkeri & 20 & $\mathrm{P}$ & Pomadasys nitidus & 22 & $\mathrm{D}$ \\
\hline Eucinostomus gracilis & 7 & $\mathrm{D}$ & Cyclopsetta panamensis & 16 & $\mathrm{D}$ & Etropus crossotus & 13 & $\mathrm{D}$ \\
\hline Etropus crossotus & 5 & $\mathrm{D}$ & Etropus crossotus & 7 & $\mathrm{D}$ & Diapterus peruvianus & 12 & $\mathrm{D}$ \\
\hline Stellifer fuerthii & 5 & $\mathrm{D}$ & Symphurus melanurus & 6 & $\mathrm{D}$ & Selene peruviana & 9 & B \\
\hline Pomadasys nitidus & 3 & $\mathrm{D}$ & Stellifer illecebrosus & 5 & $\mathrm{D}$ & Eucinostomus gracilis & 8 & $\mathrm{D}$ \\
\hline \multicolumn{9}{|c|}{ Biomass } \\
\hline Winter 2001-2002 & $\%$ & $\mathrm{H}$ & Spring 2002 & $\%$ & $\mathrm{H}$ & Spring 2004 & $\%$ & $\mathrm{H}$ \\
\hline Eucinostomus entomelas & 19 & $\mathrm{D}$ & Sphoeroides annulatus & 10 & $\mathrm{D}$ & Selene peruviana & 22 & B \\
\hline Sphoeroides annulatus & 12 & $\mathrm{D}$ & Rhinobatos glaucostigma & 5 & $\mathrm{D}$ & Sphoeroides annulatus & 11 & $\mathrm{D}$ \\
\hline Urotrygon chilensis & 8 & $\mathrm{D}$ & Eucinostomus entomelas & 5 & $\mathrm{D}$ & Rhinobatos glaucostigma & 7 & $\mathrm{D}$ \\
\hline Albula nemoptera & 5 & $\mathrm{D}$ & Menticirrhus elongatus & 4 & $\mathrm{D}$ & Eucinostomus gracilis & 6 & $\mathrm{D}$ \\
\hline Menticirrhus elongatus & 5 & $\mathrm{D}$ & Albula vulpes & 4 & $\mathrm{D}$ & Stellifer fuerthii & 5 & $\mathrm{D}$ \\
\hline Summer 2004 & $\%$ & $\mathrm{H}$ & Spring 2005 & $\%$ & $\mathrm{H}$ & Summer 2005 & $\%$ & $\mathrm{H}$ \\
\hline Stellifer fuerthii & 13 & $\mathrm{D}$ & Sphoeroides annulatus & 16 & $\mathrm{D}$ & Pomadasys nitidus & 17 & $\mathrm{D}$ \\
\hline Pomadasys nitidus & 10 & $\mathrm{D}$ & Cyclopsetta panamensis & 13 & $\mathrm{D}$ & Diapterus peruvianus & 16 & $\mathrm{D}$ \\
\hline Diapterus peruvianus & 8 & $\mathrm{D}$ & Urotrygon chilensis & 7 & $\mathrm{D}$ & Rhinobatos glaucostigma & 6 & $\mathrm{D}$ \\
\hline Pomadasys branickii & 6 & $\mathrm{D}$ & Pomadasys panamensis & 7 & $\mathrm{D}$ & Gymnura marmorata & 6 & $\mathrm{D}$ \\
\hline Cynoscion stolzmanni & 5 & $\mathrm{D}$ & Urotrygon nana & 6 & $\mathrm{D}$ & Etropus crossotus & 5 & $\mathrm{D}$ \\
\hline
\end{tabular}

D: demersal; B: benthopelagic; P: pelagic. 
TABLE 3: Results obtained when fitting the log normal distribution to our data and results of the chi-squared tests of the observed and expected observations.

\begin{tabular}{lccccccccccc}
\hline & Dec. 02 & Jan. 02 & Feb. 02 & Mar. 02 & Apr. 02 & May. 02 & May. 04 & Jun. 04 & July. 04 & Mar. 05 & Jun. 05 \\
\hline Obs. $\log _{10} M$ & 0.81 & 0.76 & 0.76 & 0.88 & 0.93 & 1.12 & 1.77 & 1.37 & 1.11 & 1.71 \\
Obs. $\log _{10} S^{2}$ & 0.32 & 0.48 & 0.42 & 0.35 & 0.36 & 0.33 & 0.64 & 0.16 & 0.37 & 0.56 & 0.48 \\
Est. $\log _{10} M$ & 0.73 & 0.44 & 0.57 & 0.8 & 0.86 & 1.08 & 1.73 & 1.37 & 1.05 & 1.69 & 1.98 \\
Est. $\log _{10} S^{2}$ & 0.4 & 0.82 & 0.62 & 0.45 & 0.45 & 0.39 & 0.73 & 0.16 & 0.45 & 0.61 & 0.48 \\
Total pred. sp. & 67.4 & 106.06 & 92.32 & 111.55 & 111.63 & 81.05 & 104.9 & 31 & 32.7 & 54.3 & 49.03 \\
Total obs. sp. & 64 & 84 & 80 & 106 & 107 & 80 & 104 & 31 & 32 & 54 & 49 \\
$\lambda$ diversity & 107.03 & 117.04 & 117.03 & 167.03 & 166.73 & 130.6 & 123.05 & 76.85 & 48.96 & 69.31 & 70.48 \\
$\chi 2$ & 2.59 & 3.94 & 9.13 & 9.8 & 5.9 & 7.95 & 2.15 & 1.78 & 7.62 & 4.3 & 13.22 \\
D. F. & 6 & 8 & 9 & 8 & 6 & 7 & 12 & 6 & 8 & 10 & 11 \\
$P$ value & 0.86 & 0.86 & 0.43 & 0.28 & 0.43 & 0.34 & 0.71 & 0.94 & 0.47 & 0.93 & 0.28 \\
\hline
\end{tabular}

Obs.: observed; est.: estimated; pred.: predicted; sp.: species; $M$ : mean; $S^{2}$ : variance; D. F.: degrees of freedom.

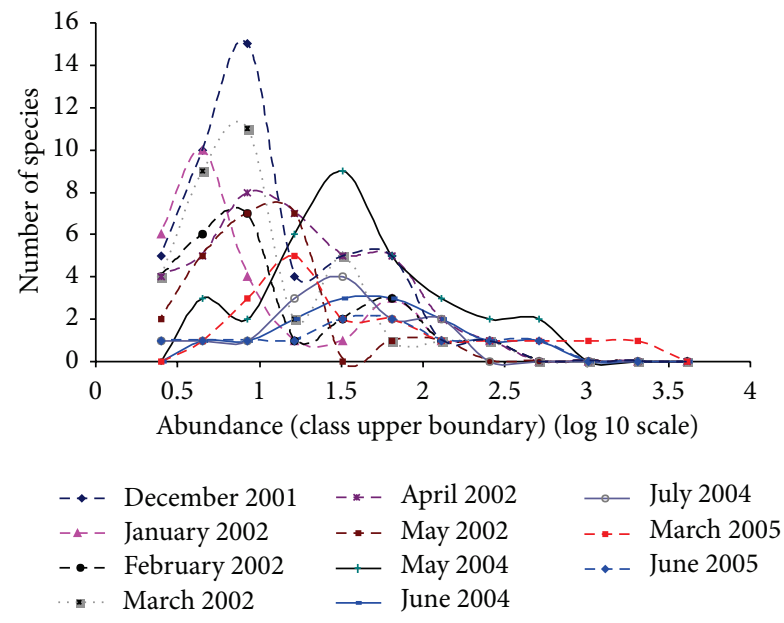

FIGURE 4: Frequency of species in relation to abundance in the different sampling months.

is not present as is the case of the plots from 2001 to 2002 . The months of 2001 and 2002 were characterized by having many species with fewer individuals, as opposed to the other years in which less species were found but with more individuals.

The Simpson's diversity index varied from 1.21 during May 2004 to 3.56 during April 2002. Comparisons between months in different years were in general not possible, but March and May 2002 showed higher values of diversity than during the same months in the following sampled years (Figure 5). Diversity decreased from December 2001 to February 2002 and increased during spring 2002. During 2004 diversity was generally lower with the exception of June 2004 when it was around 2.8, which is similar to spring 2002. During 2005, the diversity was 2.45 in March and 2.32 in June and showed the same trend observed during spring 2002, but with lower values.

Season and year influenced the arrangement of the fish assemblage, and groups were formed according to these two factors (MDS plot, stress $=0.14$ ) (Figure 6); data from the different seasons was grouped together, as well as data from the same years. These groups were corroborated by the

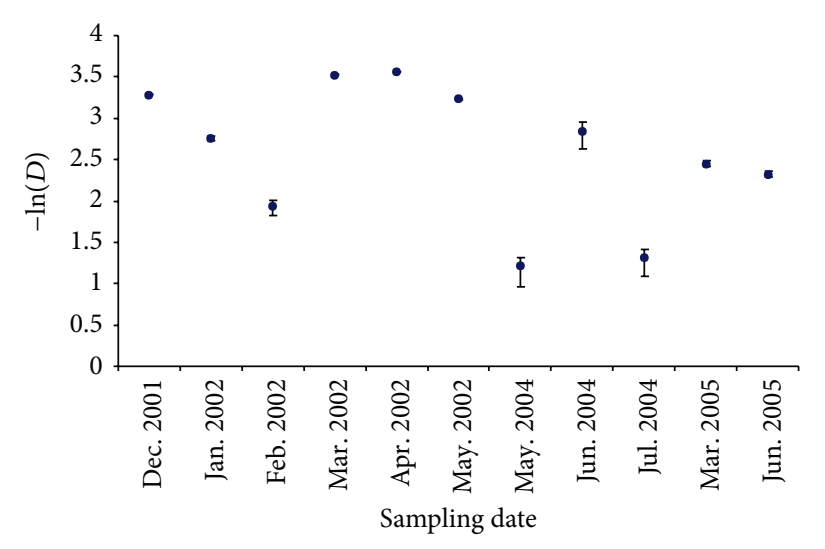

Figure 5: Values of the Simpson index $(-\ln D)$ in the different sampled seasons (confidence interval 95\%).

ANOSIM; data from winter was significantly different from that of spring $(R$-statistic $=0.778, P<0.1)$ and summer $(R$ statistic $=0.878, P<0.1)$, with no significant differences between spring and summer seasons $(R$-statistic $=0.0, P>$ $0.5)$. The data from 2002 was significantly different from the data of $2004(R$-statistic $=0.985, P<0.1)$ and $2005(R$ statistic $=0.979, P<0.1)$, with no differences found between 2004 and $2005(R$-statistic $=0.002, P>0.5)$. The species responsible for these differences varied seasonally. SIMPER results indicated that Opisthopterus dovii and Peprilus medius were more abundant during spring, and E. currani and Sphoeroides lobatus were more abundant during summer than winter. In terms of annual differences, E. crossotus was more abundant during 2004-2005 than during 2002, E. gracilis was more abundant during 2004, and $P$. nitidus was more abundant during 2005 than during 2002.

\section{Discussion}

To our knowledge, this is the most comprehensive study of the ichthyofauna in a coastal lagoon in the Gulf of California. From our results, it can be considered to be a representative description of the general composition of juveniles and adult 


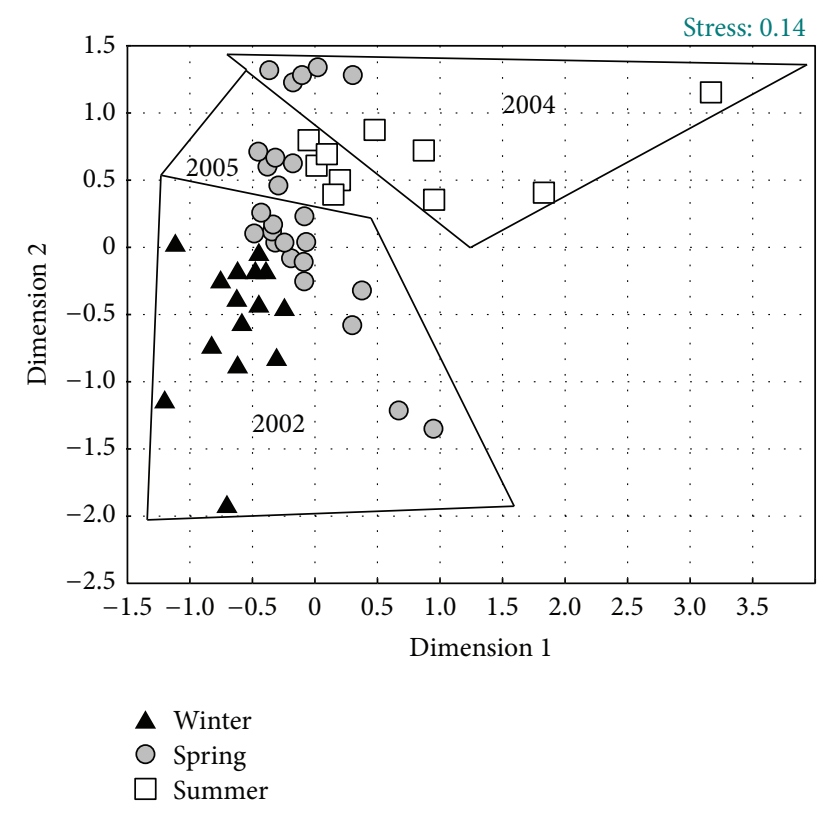

FIGURE 6: MDS plot showing the ordination of the samples according to season and year.

fish inhabiting this system. Previous studies have not been undertaken in this area; therefore comparisons with previous work cannot be made. Neither is possible to determine if the total number of species has increased or decreased.

The total number of fish species in the system remains speculative but we consider the estimate of 191 species found in this study close to the total number of species that inhabited the system during the sampling period, and only rare species were not captured. But considering that the data fitted to a log normal distribution we can conclude that our sample size was adequate in all months which allow us to unveil this distribution [32].

We are confident that we captured the majority of the fish species in the system because the three types of fishing gears that we used permitted us to capture a wide range of lengths. Although the catchability is likely to vary with size within and between species, the use of these three gears, which operate in different parts of the water column and with different mesh sizes, allowed us to capture fish from 3 to $103 \mathrm{~cm}$. The shrimp trawl net catches demersal and benthopelagic species from small to large, the gill net captures usually large size benthopelagic species although small individuals can also be captured, and the suripera net captures small demersal and benthopelagic fish species because of its reduced mesh size.

Amezcua et al. [28] also concluded that these three gears catch fish from the entire water column as well as individuals of a wide range of sizes. A reason we might have missed species in our sampling is that our sampling program was not continuous. We are missing information from 2003 and various seasons of the other years included in the study. We could have also increased the number of species captured by increasing the survey periods, so that diel activities were accounted for.
Studies of this kind in similar systems in the eastern Gulf of California are scarce but similar systems elsewhere in the region might present a similar diversity of species. Balart et al. [22] report 109 species in Ohuira, Topolobampo, and Santa María lagoons, which are situated north of the area of our study. In addition, Chan Gonzalez [41] reports 55 species from El Verde, Amezcua-Linares [42] reports 60 species in Huizache-Caimanero, and Alvarez-Rubio et al. [43] report 76 species in Teacapán-Agua Brava. These systems are located south of our study site. Balart et al. [22] and Chan Gonzalez [41] analyzed commercial catches to produce a list of species and did not include effort as variable. Amezcua-Linares [42] and Alvarez-Rubio et al. [43] sampled approximately 100 stations during a one-year period using three fishing gears at each station: a trawl net, a gill net, and a seine net. The fishing gears they used are similar to the ones we used in our study, but the sampling effort in our study was higher since we managed to sample more than 1500 stations during the period of our study. If the previous studies had used effort similar to that of this study, the number of species found in those studies might have been higher. Unfortunately, the previous studies did not include species accumulation curves or other analyses that would give an idea of the total number of species, so we are unable to determine if the number of species they found is close to the potential total or not.

The majority of the fish species inhabiting the system are demersal, although in most seasons pelagic or benthopelagic species also showed high abundances. The genus Selene spp. was highly abundant in most seasons and during the year 2004. It is known that this species is a common resident in these systems [44], but an explanation of its increased abundance during 2004 cannot be given. During spring 2002 and 2005 this species was not amongst the most abundant species, but the pelagic species Pliosteostoma lutipinnis and A. macrolepidota during 2002 and A. walkeri were very abundant during 2004. Castro-Aguirre et al. [44] report the entrance of these species to the estuarine system as a common behavior apparently associated with the temperature of the sea water at those times. The reason that these small pelagic species were not very abundant during spring 2004 might be the high numbers of S. peruviana that were already occupying the habitat and therefore precluding a high abundance of other species. This would indicate that the pelagic habitat in the estuarine habitats is a limiting factor as opposed to the demersal one, but further research is necessary to test this hypothesis.

The fish assemblage of the lagoon system of Santa Maria la Reforma showed annual and seasonal variations. This was observed in the contrasting values of diversity $\left(D^{\prime}\right)$ and the groups formed using multivariate analyses. The diversity changed monthly indicating changes in the species composition. These changes in diversity help explain the species accumulation curve in which the number of species increased after reaching an asymptote in certain months, indicating that the fish assemblage changed seasonally, with new species arriving the system through the year. These increases in the number of species coincide with increases in the diversity index and in the number of new species, which occurred from February to March 2002, indicating the arrival of more 
species that had not been recorded previously. Similar results occurred in June 2004 and March 2005.

These results indicate that the lagoon system is used by a wide variety of fish species but that the use by each species differs through the year depending on their ecology, for example, the formation of spawning aggregations or migration behavior of larger fish migrating out of the system as they grow.

The multivariate results clearly indicate a seasonal transition through the year, which seems to be related to seasonal migration patterns of the fish fauna, with a diversity that varies as some fish species leave the system, which could explain the decrease in the diversity, and others arrive it, which could explain the suddenly increase in the diversity, pointing to a differential use of this system by the different fish species; however further research is needed to corroborate this assumption, but it might be possible that this is occurring considering that previous studies have reported that these kind of systems show a high seasonal stability and adaptation of the species to variations in temperature, where seasonal patterns are maintained even during warming events [45-47].

Our results also show annual variations in the fish assemblage of the studied system, which was different from 20012002 to 2004-2005. The reason for the annual changes could be related to the timing of our samples, since during the years 2001-2002 most of the sampling was undertaken during the winter and spring, and during the following years most of the sampling was undertaken during summer, so when the years are compared, the differences could be a result of seasonal differences rather than annual ones.

Our study clearly shows a seasonal succession in the fish assemblage in the system and it leaves the hypothesis that these changes could be related to a partitioning of the habitat by the different species using the habitat through the year. It is necessary to consider that the temperature shows considerable fluctuations through the year, so this factor might also be important in determining the composition of the fish assemblage. Changes in abundance and species composition occur frequently in fish communities sharing neighboring biogeographical areas, as a result of migratory movements related to climate and oceanographic changes. The area of our study is a transition zone between the ichthyofauna of the Mexican province, which goes from the Gulf of Tehuantepec to Topolobampo (north of the studied area), and the Gulf of California province, that extends from Topolobampo to the north [48]. In this sense, the fish assemblage found during winter could be representatives of the Gulf of California province, whilst the fish assemblage present during summer could be representative of the Mexican province, with transitions between these seasons, but a detailed analysis of the distribution of the species present in each season is needed to corroborate this assumption.

\section{Conflict of Interests}

The authors declare that there is no conflict of interests regarding the publication of this paper.

\section{Acknowledgments}

The National Institute of Fisheries (INAPESCA), through the Regional Centre of Fisheries Research in Mazatlan (CRIPMazatlan), proportionated the authors with samples for this study. This work was funded by the Research Project PAPIIT IN208911.

\section{References}

[1] S. J. M. Blaber and T. G. Blaber, "Factors affecting the distribution of juvenile estuarine and inshore fish," Journal of Fish Biology, vol. 17, pp. 143-162, 1980.

[2] R. C. J. Lenanton and I. C. Potter, "Contribution of estuaries to commercial fisheries in temperate Western Australia and the concept of estuarine dependence," Estuaries, vol. 10, no. 1, pp. 28-35, 1987.

[3] P. Laegdsgaard and C. R. Johnson, "Mangrove habitats as nurseries: unique assemblages of juvenile fish in subtropical mangroves in eastern Australia," Marine Ecology Progress Series, vol. 126, no. 1-3, pp. 67-81, 1995.

[4] M. Barletta, A. Barletta-Bergan, U. Saint-Paul, and G. Hubold, "Seasonal changes in density, biomass, and diversity of estuarine fishes in tidal mangrove creeks of the lower Caeté Estuary (northern Brazilian coast, east Amazon)," Marine Ecology Progress Series, vol. 256, pp. 217-228, 2003.

[5] A. Yáñez-Arancibia, L. Lara-Domínguez, A. Aguirre-León et al., "Ecology of dominant fish population on tropical estuaries: environmental factors regulating biological strategies and production," in Fish Community Ecology in Estuaries and Coastal Lagoons: Towards an Ecosystem Integration, A. YáñezArancibia, Ed., pp. 311-366, Universitaria, UNAM. PUALICML, Mexico City, Mexico, 1985.

[6] S. M. Warlen, K. W. Able, and E. H. Laban, "Recruitment of larval Atlantic menhaden (Brevoortia tyrannus) to North Carolina and New Jersey estuaries: evidence for larval transport northward along the east coast of the United States," Fishery Bulletin, vol. 100, no. 3, pp. 609-623, 2002.

[7] D. Pauly, "Fisheries research and the demersal fisheries of Southeast Asia," in Fish Population Dynamics, J. A. Gulland, Ed., pp. 329-348, John Wiley \& Sons, New York, NY, USA, 1988.

[8] D. Pauly and A. Yáñez-Arancibia, "Fisheries in Coastal Lagoons," in Coastal Lagoon Processes, B. Kjerfve, Ed., pp. 377399, Elsevier, Amsterdam, The Netherlands, 1994.

[9] M. Barletta, A. Barletta-Bergan, and U. Saint-Paul, "Description of the fishery structure in the mangrove dominated region of Bragança (State of Para-North Brazil)," Ecotropica, vol. 4, pp. 4153, 1998.

[10] S.-R. Kuo, H.-J. Lin, and K.-T. Shao, "Seasonal changes in abundance and composition of the fish assemblage in Chiku Lagoon, southwestern Taiwan," Bulletin of Marine Science, vol. 68, no. 1, pp. 85-99, 2001.

[11] S. J. M. Blaber, D. P. Cyrus, J.-J. Albaret et al., "Effects of fishing on the structure and functioning of estuarine and nearshore ecosystems," ICES Journal of Marine Science, vol. 57, no. 3, pp. 590-602, 2000.

[12] S. A. Shepherd, A. J. McComb, D. A. Bulthuis, V. Neverauskas, and D. A. R. W. Steffenson, "Decline of sea grasses," in Biology of Sea Grasses, A. W. D. Larkum, A. J. McComb, and S. 
A. Shepherd, Eds., pp. 346-392, Elsevier, Amsterdam, The Netherlands, 1989.

[13] J. L. McHugh, “The estuarine ecosystem integrated," in Fish Community Ecology in Estuaries and Coastal Lagoons: Towards an Ecosystem Integration, A. Yanez-Arancibia, Ed., pp. 9-15, Universitaria, UNAM. PUAL-ICML, Mexico City, Mexico, 1985.

[14] S. A. Harris, D. P. Cyrus, and L. E. Beckley, "Horizontal trends in larval fish diversity and abundance along an ocean-estuarine gradient on the northern KwaZulu-Natal coast, South Africa," Estuarine, Coastal and Shelf Science, vol. 53, no. 2, pp. 221-235, 2001.

[15] W. J. Kimmerer, J. Cowan J.H., L. W. Miller, and K. A. Rose, "Analysis of an estuarine striped bass population: effects of environmental conditions during early life," Estuaries, vol. 24, no. 4 , pp. 557-575, 2001.

[16] S. G. Ayvazian and G. A. Hyndes, "Surf-zone fish assemblages in south-western Australia: do adjacent nearshore habitats and the warm Leeuwin current influence the characteristics of the fish fauna?" Marine Biology, vol. 122, no. 4, pp. 527-536, 1995.

[17] G. A. Hyndes, I. C. Potter, and S. A. Hesp, "Relationships between the movements, growth, age structures, and reproductive biology of the teleosts Sillago burrus and S. vittata in temperate marine waters," Marine Biology, vol. 126, no. 3, pp. 549-558, 1996.

[18] G. A. Hyndes, I. C. Potter, and R. C. J. Lenanton, "Habitat partitioning by whiting species (Sillaginidae) in coastal waters," Environmental Biology of Fishes, vol. 45, no. 1, pp. 21-40, 1996.

[19] J. Dulčić and B. Glamuzina, "Length-weight relationships for selected fish species from three eastern Adriatic estuarine systems (Croatia)," Journal of Applied Ichthyology, vol. 22, no. 4, pp. 254-256, 2006.

[20] G. W. Thayer, D. R. Colby, and W. F. Hettler, "Utilization of the red mangrove prop root habitat by fishes in south Florida," Marine Ecology Progress Series, vol. 35, pp. 25-38, 1987.

[21] A. Yåñez-Arancibia, A. L. Lara-Dominguez, J. L. Rojas-Galaviz, P. Sånchez-Gil, J. W. Day Jr., and C. J. Madden, "Seasonal biomass and diversity of estuarine fishes coupled with tropical habitat heterogeneity (southern Gulf of Mexico)," Journal of Fish Biology, vol. 33, no. 1, pp. 191-200, 1988.

[22] E. F. Balart, J. L. Castro-Aguirre, and R. Torres-Orozco, "Ictiofauna de las bahìas de Ohuira, Topolobampo y Santa María, Sinaloa, México," Investigaciones Marinas, vol. 79, no. 2, pp. 91103, 1992.

[23] B. Aguilar-Palomino, C. Pérez Reyes, F. Galván-Magaña, and L. A. Abitía-Cardenas, "Ictiofauna de la Bahía de Navidad, Jalisco, México," Revista de Biologia Tropical, vol. 49, no. 1, pp. 173-190, 2001.

[24] M. Wolff, "Demersal fish assemblages along the Pacific coast of Costa Rica: a quantitative and multivariate assessment based on the Victor Hensen Costa Rica Expedition (1993/1994)," Revista de Biologia Tropical, vol. 44, no. 3, pp. 187-214, 1996.

[25] S. J. M. Blaber and D. A. Milton, "Species composition, community structure and zoogeography of fishes of mangrove estuaries in the Solomon Islands," Marine Biology, vol. 105, no. 2, pp. 259267, 1990.

[26] P. A. Ramey and P. V. R. Snelgrove, "Spatial patterns in sedimentary macrofaunal communities on the south coast of Newfoundland in relation to surface oceanography and sediment characteristics," Marine Ecology Progress Series, vol. 262, pp. 215-227, 2003.

[27] W. Fischer, F. Krupp, W. Schneider, C. Sommer, K. E. Carpenter, and V.H. Niem, Guía FAO para la Identificación de Especies para los Fines de la Pesca: Pacífico centro-oriental, FAO, Rome, Italy, 1995.

[28] F. Amezcua, J. Madrid-Vera, and H. Aguirre-Villaseñor, "Effect of the artisanal shrimp fishery on the ichthyofauna in the coastal lagoon of Santa María la Reforma, southeastern Gulf of California," Ciencias Marinas, vol. 32, no. 1B, pp. 97-109, 2006.

[29] P. Sparre and S. C. Venema, Introduction toTropical Fish Stock Assessment. Part 1, FAO, Rome, Italy, 1998.

[30] M. Haddon, Modelling and Quantitative Methods in Fisheries, Chapman and Hall/CRC, Boca Raton, Fla, USA, 2001.

[31] C. H. Flather, "Fitting species-accumulation functions and assessing regional land use impacts on avian diversity," Journal of Biogeography, vol. 23, no. 2, pp. 155-168, 1996.

[32] A. E. Magurran, Measuring Biological Diversity, Blackwell, Oxford, UK, 2004.

[33] B. Efron and R. Tibshirani, An Introduction to the Bootstrap, Chapman and Hall, New York, NY, USA, 1993.

[34] E. C. Pielou, Ecological Diversity, John Wiley \& Sons, New York, NY, USA, 1975.

[35] R. R. Sokal and F. J. Rholf, Biometry, WH Freeman, San Francisco, Calif, USA, 1969.

[36] M. L. Rosenzweig, Species Diversity in Space and Time, Cambridge University Press, Cambridge, UK, 1995.

[37] K. R. Clarke, "Non-parametric multivariate analyses of changes in community structure," Australian Journal of Ecology, vol. 18, no. 1, pp. 117-143, 1993.

[38] N. R. Collins and R. Williams, "Zooplankton communities in the Bristol Channel and Severn Estuary," Marine Ecology Progress Serie, vol. 9, pp. 1-11, 1982.

[39] K. R. Clarke and R. M. Warwick, Change in Marine Communities: An Approach to Statistical Analysis and Interpretation, Plymouth, 1994.

[40] StatSoft Inc., "STATISTICA (data analysis software system), version 6," 2001, http://www.statsoft.com/.

[41] R. Chan-Gonzalez, Composición y abundancia de la ictiofauna del estero el Verde, Sinaloa, Centro Interdisciplinario de Ciencias Marinas, Instituto Politécnico Nacional, Mexico City, Mexico, 1980.

[42] F. Amezcua-Linares, "Generalidades ictiológicas del sistema lagunar costero Huizache-Caimanero, Sinaloa, México," Anales del Instituto de Ciencias del Mar y Limnología, vol. 4, pp. 1-26, 1977.

[43] M. Alvarez-Rubio, F. Amezcua-Linares, and A. YañezArancibia, "Ecología y estructura de las comunidades de peces en el sistema lagunar Teacapán-Agua Brava, México," Anales del Instituto de Ciencias del Mar y Limnología, vol. 13, pp. 185-242, 1986.

[44] J. L. Castro-Aguirre, H. Espinosa Peréz, and J. J. Schmitter-Soto, Ictiofauna estuarino-lagunar y vicaria de México, LIMUSA, Mexico City, Mexico, 1999.

[45] R. Palomares-García and J. Gómez-Gutiérrez, "Copepod community structure at Bahia Magdalena, Mexico during El Niño 1983-84," Estuarine, Coastal and Shelf Science, vol. 43, no. 5, pp. 583-595, 1996. 
[46] J. Gómez-Gutiérrez, R. Palomares-García, R. De Silva-Dávila, M. A. Carballido-Carranza, and A. Martínez-López, "Copepod daily egg production and growth rates in Bahia Magdalena, Mexico," Journal of Plankton Research, vol. 21, no. 12, pp. 22272244, 1999.

[47] R. Avendaño-Ibarra, R. Funes-Rodríguez, A. Hinojosa-Medina, R. González-Armas, and G. Aceves-Medina, "Seasonal abundance of fish larvae in a subtropical lagoon in the west coast of the Baja California Peninsula," Estuarine, Coastal and Shelf Science, vol. 61, no. 1, pp. 125-135, 2004.

[48] F. Amezcua-Linares, Peces demersales del Pacífico de México, Instituto de Ciencias del Mar y Limnología-Universidad Nacional Autónoma de México, Mexico City, Mexico, 2009. 

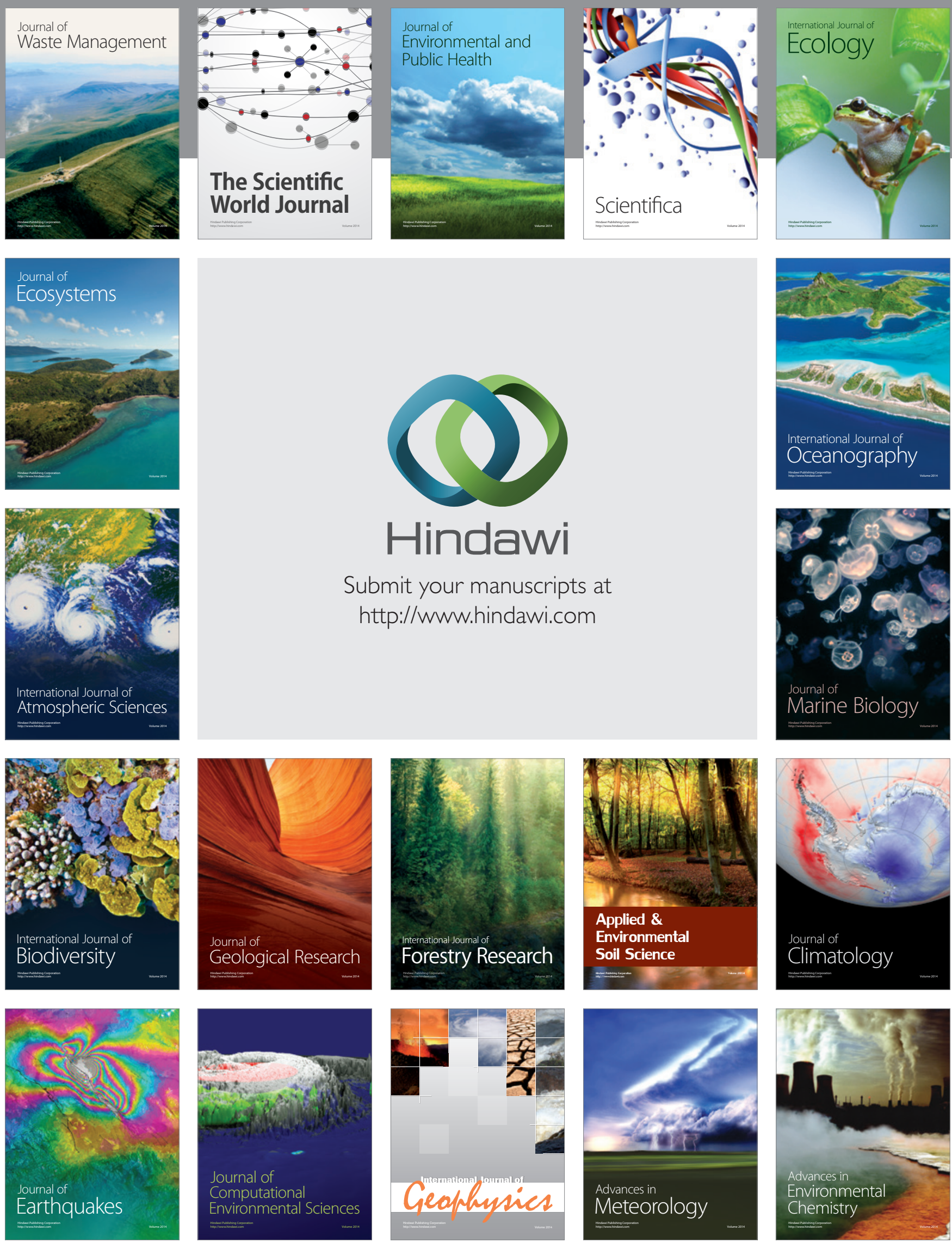